\title{
Isolated hypophosphataemia as an early marker of primary hyperparathyroidism
}

\author{
John Alexander and Dinesh Nagi \\ Endocrinology and Diabetes, Mid-Yorkshire NHS Trust, Wakefield, England
}

Correspondence

should be addressed

to J Alexander

Email

jonealexander@gmail.com

\section{Summary}

Primary hyperparathyroidism (PHPT) is a disease caused by overactive parathyroid glands with consequent hypercalcaemia. The main cause in $85-90 \%$ of the cases is the presence of a solitary parathyroid adenoma. The most common presentation is with asymptomatic hypercalcaemia diagnosed on routine biochemical testing. Although low serum phosphate levels are an associated finding in primary hyperparathyroidism, the diagnostic criteria for PHPT remain to be hypercalcaemia, high or inappropriately normal PTH and hypercalciuria. This case report presents a patient who presented with low phosphate levels without any other biochemical evidence of PHPT, who returned several years later with overt primary hyperparathyroidism. This report intends to raise interest among the medical fraternity whether there is a need to consider hypophosphataemia as an early sign of PHPT.

\section{Learning points}

- Primary hyperparathyroidism is a relatively common condition with varying clinical and biochemical presentation.

- The most common presentations still remain as an asymptomatic biochemical abnormality closely related to calcium, PTH and bone metabolism.

- Not much attention is usually given to associated biochemical abnormalities, and hence they are usually less investigated.

- Further research is needed to establish if patients need long-term monitoring when no obvious cause for isolated hypophosphataemia has been found.

\section{Background}

The biochemical definition of primary hyperparathyroidism is hypercalcaemia with elevated or inappropriately normal parathyroid hormone (PTH) levels secondary to parathyroid overactivity (1). Primary Hyperparathyroidism (PHPT) occurs most commonly in individuals over 50 years of age and in postmenopausal women, showing a prevalence of about $0.78 \%$ in patients evaluated in reference services $(3,4)$. The most common presentation is asymptomatic hypercalcaemia detected on routine screening, although the clinical presentation is highly variable (2). Low phosphate is usually seen along with the above findings in PHPT, especially in severe forms of the disease (5). We describe a unique clinical encounter where the low phosphate was the only biochemical finding in a patient who presented several years later with overt primary hyperparathyroidism.

\section{Case presentation}

A 67-year-old gentleman was referred to the Endocrine department as he was incidentally found to have a low phosphate in April 2014. Patient had some abdominal discomfort lasting for a few weeks, and this prompted the general practitioner to arrange the biochemical assessments, which showed a phosphate of $0.66 \mathrm{mmol} / \mathrm{L}$ (0.8-1.5 mmol/L). His adjusted calcium level was normal 
at $2.52 \mathrm{mmol} / \mathrm{L}$ along with normal albumin $(38 \mathrm{~g} / \mathrm{L})$ and alkaline phosphatase $(47 \mathrm{U} / \mathrm{L})$. He also underwent an ultrasound scan of the liver, which was reported as normal. His symptoms resolved spontaneously and no other causes for his symptoms were found, and he was then referred to Endocrinology department for further assessment of his hypophosphataemia.

His past medical history includes systemic lupus erythrematosis with positivity for La and Ro antibodies and was in remission at the time of the review. He also had the previous history of myositis, peripheral neuropathy, gastro-oesophageal reflux disease, benign hyperplasia of the prostate, Iron deficiency anaemia and viral keratitis (treated). His regular medications include azathioprine $100 \mathrm{mg}$ once a day (OD), rabeprazole $40 \mathrm{mg}$ OD, aspirin $75 \mathrm{mg}$ OD, tamsulosin $400 \mu \mathrm{g}$ OD and Ferrous sulphate 200mg OD. He was an ex-coal miner and a nonsmoker, and he drinks two bottles of wine per week.

After his review in the Endocrine clinic in April 2014, he remained completely asymptomatic. There were no symptoms suggestive of hypercalcaemia, and he denied any history of previous fractures or osteoporosis. No symptoms of hypophosphataemia such as myalgia, bone pain or muscle weakness were reported on evaluation. There was no clinical suggestion of any dietary deficiency. There was no family history of any electrolyte abnormalities or any skeletal deformities to suggest any inborn errors of metabolism.

On examination, he weighed $87.9 \mathrm{~kg}$, and his height was $1.72 \mathrm{~m}$. His pulse rate was 91 b.p.m., and his BP was 105/75 mmHg. His systemic examination was unremarkable, and his skeletal system examination did not reveal any abnormalities. His proximal and distal muscle strength were normal, and there were no abnormal neurological signs to elicit. He had a repeat biochemical assessment done which showed a phosphate of $0.60 \mathrm{mmol} / \mathrm{L}$ with a normal adjusted calcium of 2.56 $\mathrm{mmol} / \mathrm{L}(2.2-2.6 \mathrm{mmol} / \mathrm{L})$ and normal PTH $(5.8 \mathrm{pmol} / \mathrm{L}$ - normal range 1.6-6.9 pmol/L). His vitamin D level was $57.1 \mathrm{nmol} / \mathrm{L}$ (>50 nmol/L suggests vitamin D sufficiency). Since there were no suggestions of any other serious causes of hypophosphataemia, the patient was discharged from secondary care without any prescription for any phosphate supplements.

He was rereferred to the secondary care in July 2019 with hypercalcaemia, raised PTH and low phosphate. His past medical conditions remained the same as in 2014. His regular medications included azathioprine $50 \mathrm{mg}$ OD, ferrous sulphate $200 \mathrm{mg} \mathrm{BD}$, lansoprazole $30 \mathrm{mg} \mathrm{OD}$, and tamsulosin $400 \mu \mathrm{g}$ OD. This time he reported constipation, depression and polyuria which were attributed to hypercalcaemia. Clinical examination was unremarkable with normal observations and his weight remained the same as 2014. There was no skeletal deformity identified. He did not sustain any fractures since 2014 .

\section{Investigation}

His biochemical assessment showed a phosphate of 0.41 $\mathrm{mmol} / \mathrm{L}$, adjusted calcium of $2.96 \mathrm{mmol} / \mathrm{L}$ and a PTH of $10.6 \mathrm{pmol} / \mathrm{L}$. His vitamin D level was in the sufficient range $(52.7 \mathrm{nmol} / \mathrm{L})$. He underwent further investigations with a $24 \mathrm{~h}$ urine calcium collection which was reported as normal $(5.7 \mathrm{mmol} /$ day, normal range $2.5-7.5 \mathrm{mmol} /$ day $)$ with a raised urinary calcium clearance ratio of 0.0149 . His renal functions remained normal with an eGFR of $78 \mathrm{~mL} / \mathrm{min} / 1.73 \mathrm{~m}^{2}$. His magnesium and alkaline phosphatase levels were within normal limits. His liver functions were also normal. All other biochemical investigations of hypercalcaemia were negative and there were no suggestions of malignancy. His USS scan of the kidneys were normal and his bone density scan showed osteopenia at the femoral neck (T score of -1.2).

The USS scan of the neck did not identify any parathyroid adenoma but incidentally found a U3 nodule in the left thyroid lobe. He subsequently underwent a SPECT CT scan which identified a Sestamibi positive right inferior parathyroid adenoma. He also had a fine needle biopsy of his thyroid nodule which revealed atypical cells and was pathologically classified as Thy3 (Royal College of Pathologists guidance on Thyroid cytology).

\section{Treatment}

A reliable diagnosis of primary hyperparathyroidism was made and due to his symptoms of hypercalcaemia. He was started on Cinalcalcet 30mg BD and his adjusted calcium levels returned to normal $(2.43 \mathrm{mmol} / \mathrm{L})$ although his phosphate levels remained below the normal level $(0.65 \mathrm{mmol} / \mathrm{L})$. He successfully underwent right inferior parathyroidectomy and left thyroid lobectomy in November 2020 as there was considerable disruptions in the delivery of healthcare due to Covid pandemic. His immediate post-operative adjusted calcium, phosphate and PTH levels were normal $(2.51 \mathrm{mmol} / \mathrm{L}, 0.87 \mathrm{mmol} / \mathrm{L}$ and $2.4 \mathrm{pmol} / \mathrm{L}$ respectively) . His histology of the resected parathyroid specimen was consistent with parathyroid adenoma. 


\section{Outcome and follow-up}

Post-operatively his Cinacalcet was stopped, and he remains completely asymptomatic. On subsequent clinic reviews he remains well and his calcium and PTH levels are within the normal level. His phosphate levels are now in the normal range at the time of the publication $(1.02 \mathrm{mmol} / \mathrm{L})$. Since all the electrolyte disturbances have been resolved, he has been discharged from the Endocrine follow up.

\section{Discussion}

Primary hyperparathyroidism (PHPT) is a disease caused by overactive parathyroid glands with consequent hypercalcaemia (1). Usual biochemical findings found in PHPT are raised calcium with raised or inappropriately normal PTH levels. A variant of primary hyperpartahyroidism called normocalcaemic primary hyperparathyroidism exists (NPHPT), which is characterised by consistently elevated PTH levels with normal total and ionised calcium levels in the absence of any secondary causes of raised PTH (6). There appears to be a paucity of evidence to suggest that isolated hypophosphataemia in the presence of normal calcium and PTH would be considered as an early sign of primary hyperparathyroidism.

Although calcium levels are important in the homeostasis of bone, phosphate levels also plays an important role in the maintenance of skeletal strength and rigidity (7). Phosphate is also involved in a variety of processes including acid-base buffering, post receptor signaling, energy transfer and information storage and translation in DNA and RNA. The primary repository of phosphate (85\%) is in the bone, where calcium and phosphate (in hydroxyapatite) provide skeletal strength and rigidity. Outside of bone, most of the phosphate is intracellular.

Hypophosphataemia is generally defined as serum phosphate levels below $0.8 \mathrm{mmol} / \mathrm{L}$. The symptoms of hypophosphataemia are very nonspecific while majority of the patients with mild hypophosphataemia remains asymptomatic as in our case. The other reported symptoms of severe forms include myalgia, muscle weakness, fatigue and acute muscle pain. Chronic hypophosphataemia in turn can lead to osteomalacia, bone pain, and pseudo fractures. The genetic disorders usually cause prolonged hypophosphataemia which results in radiographic features including genu valgum and varum, frontal bossing, widening of the ends of long bones and short stature (8).
However, osteomalacia requires time to develop and is not present during acute hypophosphataemia.

It is important to be aware of the various hormones involved in phosphate homeostasis to better understand the concomitant hypophosphataemia seen in primary hyperparathyroidism. Phosphate is abundantly present in many foods mainly dairy products, meat, fish, whole grains and lentils. Isolated dietary deficiency is only seen in severe malnutrition as the availability of the phosphate in common foods is high and RDA allowance is only 700 $\mathrm{mg}$. In the intestine, phosphate and calcium activation is mediated by activated vitamin $\mathrm{D}$ which in turn is mediated by PTH. PTH also enhances the absorption of calcium and phosphate from the bones into the blood. But the main site of action of PTH is at the proximal convoluted tubules where phosphate reabsorption is reduced which in turn cause phosphaturia and subsequent hypophosphataemia in primary hyperparathyroidism. This is mediated via the inhibition of sodium phosphate cotransporter (type 2a and 2c). This step is also affected by fibroblast growth factor 23 (FGF 23)6 which can cause oncogenic osteomalacia and hence it is important to rule out malignancies in case of hypophosphataemia. Since the clinical implications of primary hyperparathyroidism are due to hypercalcaemia and hypercalciuria, low phosphate is not considered in its biochemical definition. There is not enough literature available in regards to the follow up of patients with isolated hypophosphataemia and no data exists if these patients may develop primary hyperparathyroidism in future. Also there is scarcity of data on how to manage patients with mild isolated hypophosphataemia in the long term.

All the causes of hypophosphataemia are described in Table 1 (9). A careful clinical evaluation is needed to look for the causes of hypophosphataemia . In addition, family history may reveal a heritable disorder, although some patients with inherited hypophosphataemia may be unaware of their family diagnosis (10). Apart from the genetic disorders, most of the acquired causes are related to drugs (5) (diuretics, toluene, streptozocin, ifosfamide, cisplatin, tetracyclines, aminoglycosides, antiretrovirals, imatinib, phosphate binders, Sevelamer, antacids containing calcium, magnesium, aluminum, bisphosphonates, mannitol, salicylate poisoning, Insulin and glucose infusion), nutritional factors (alcoholism, refeeding syndrome, vitamin D deficiency, malnutrition and malabsorption) and acute illness (hyperventilation, respiratory alkalosis). Although primary hyperparathyroidism causes hypophosphataemia, it is mild and accompanied by serum calcium derangements. 
Table 1 Causes of hypophosphataemia (11).

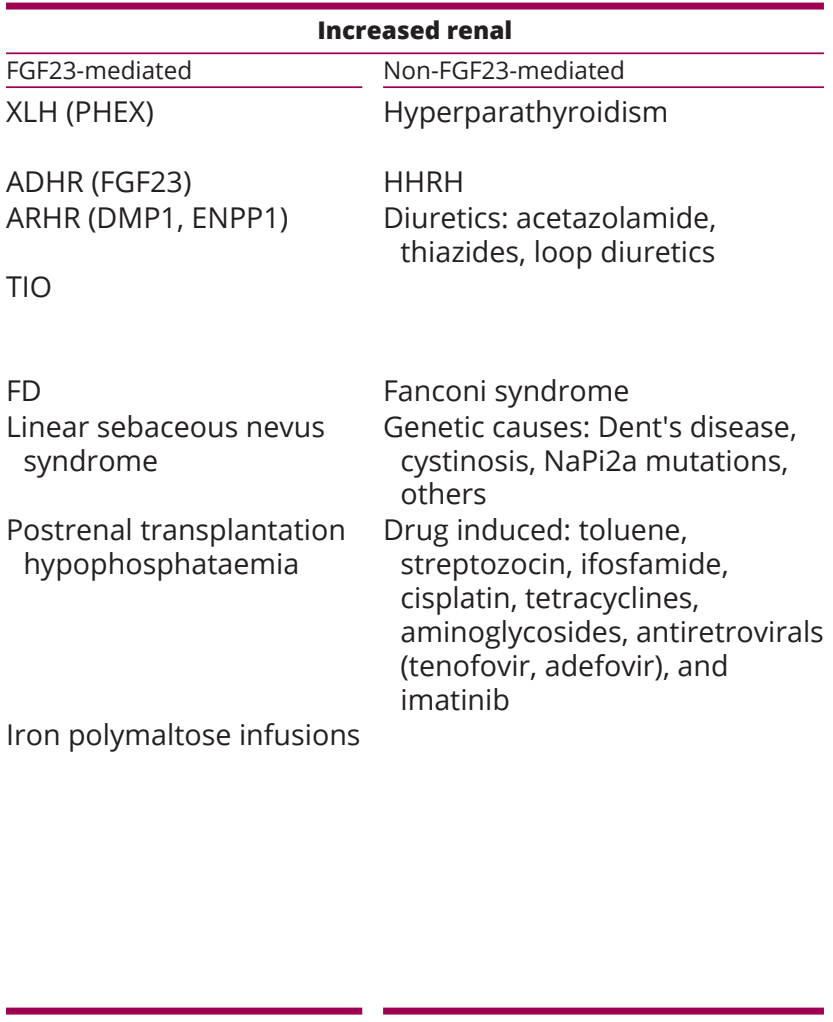

Oncogenic osteomalacia is usually accompanied by myopathy, bone pain and fracture (11).

A thorough search for the causes of hypophosphataemia from our patient did not reveal any obvious causes. This eventually led to the patient being discharged back to the primary care. There was no indication to keep the patient under biochemical monitoring. This case raises the question as to whether some patients may have an initial phase of mild hypophosphataemia in their future progression to overt PHPT. Reassuringly patient does not seem to have developed any major end organ damage in the five years of disease progress.

\section{Declaration of interest}

The authors declare that there is no conflict of interest that could be perceived as prejudicing the impartiality of the case reported.

\section{Funding}

This research did not receive any specific grant from any funding agency in the public, commercial or not-for-profit sector

\section{Patient consent}

Patient has given a written informed consent for publication of the submitted article.

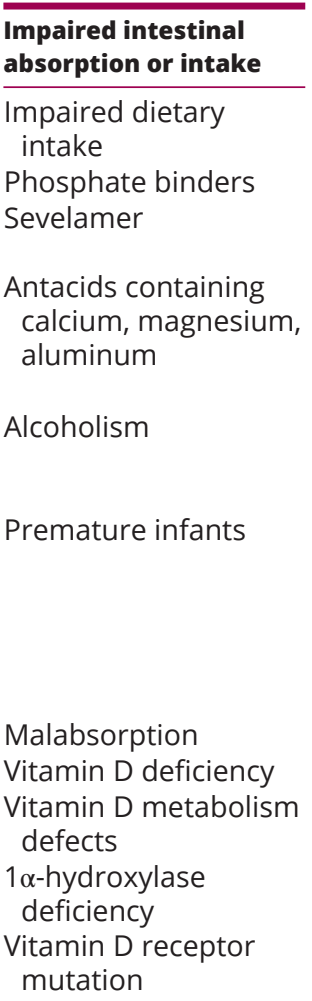

Impaired intestinal absorption or intake

Others

Refeeding syndrome Mannitol

Glucose infusion

Bisphosphonates Insulin infusion

\section{Salicylate poisoning}

Hyperventilation

Respiratory alkalosis

Catecholamines

\section{Patient's perspective}

Patient remained under the care of the joint endocrine- surgical team during treatment and has now been discharged back to primary care. He is immensely grateful to the care he has received.

\section{Author contribution statement}

$\mathrm{Dr}$ John Alexander was involved in the preparation of the manuscript, editing and submission of the case report. Dr Dinesh Nagi was the clinician involved in the management of the patient and final editing of the manuscript.

\section{References}

1 Bilezikian JP, Brandi ML, Rubin M \& Silverberg SJ. Primary hyperparathyroidism: New concepts in clinical, densitometric and biochemical features. Journal of Internal Medicine 2005257 6-17. (https://doi.org/10.1111/j.1365-2796.2004.01422.x)

2 Bandeira F, Griz L, Caldas G, Bandeira C \& Freese E. From mild to severe primary hyperparathyroidism: The Brazilian experience. Arquivos Brasileiros de Endocrinologia e Metabologia 200650 657-663. (https://doi.org/10.1590/s0004-27302006000400011)

3 Rodgers SE, Lew JI \& Solórzano CC. Primary hyperparathyroidism. Current Opinion in Oncology 200820 52-58. (https://doi.org/10.1097/ CCO.0b013e3282f2838f)

4 Eufrazino C, Veras A \& Bandeira F. Epidemiology of primary hyperparathyroidism and its non-classical manifestations in the City of Recife, brazil. Clinical Medicine Insights: Endocrinology and Diabetes 20136 69-74. (https://doi.org/10.4137/CMED.S13147)

5 Bringhurst FR. Circulating forms of parathyroid hormone: peeling back the onion. Clinical Chemistry 200349 1973-1975. (https://doi. org/10.1373/clinchem.2003.026948) 
Endocrinology,

Diabetes \& Metabolism

CASE REPORTS
John Alexander and Dinesh

Nagi
Hypophosphatemia and PHPT

ID: 20-0217; June 2021

DOI: $10.1530 / E D M-20-0217$
6 Cusano NE, Silverberg SJ \& Bilezikian JP. Normocalcemic primary hyperparathyroidism. Journal of Clinical Densitometry 2013 16 33-39. (https://doi.org/10.1016/j.jocd.2012.12.001)

7 Liamis G, Milionis HJ \& Elisaf M. Medication-induced hypophosphatemia: a review. QJM 2010103 449-459. (https://doi. org/10.1093/qjmed/hcq039)

8 Tiosano D \& Hochberg Z. Hypophosphatemia: the common denominator of all rickets. Journal of Bone and Mineral Metabolism 200927 392-401. (https://doi.org/10.1007/s00774-0090079-1)
9 Imel EA \& Econs MJ. Approach to the hypophosphatemic patient. Journal of Clinical Endocrinology and Metabolism 201297 696-706. (https://doi.org/10.1210/jc.2011-1319)

10 Econs MJ, Samsa GP, Monger M, Drezner MK \& Feussner JR. X-linked hypophosphatemic rickets: a disease often unknown to affected patients. Bone and Mineral 199424 17-24. (https://doi.org/10.1016/ s0169-6009(08)80127-4)

11 Clinkenbeard EL \& White KE. Systemic control of bone homeostasis by FGF23 signaling. Current Molecular Biology Reports 20162 62-71. (https://doi.org/10.1007/s40610-016-0035-5)

Received in final form 6 April 202

Accepted 13 May 2021 